Christakou, A., \& Lavallee, D. (2009). Rehabilitation from sports injuries: From theory to practice. Perspectives in Public Health, 129 (3), 120-126.

\begin{abstract}
A great number of injuries occur in the context of recreational physical activities and competitive athletics. Adherence to sport injury rehabilitation means injured athlete's compliance (or not) to a sports medicine/injury personnel's instructions of participating in a rehabilitation programme in a clinic, and/or doing rehabilitation exercises on home. It has emerged as an area of interest among physiotherapists and other allied professionals (i.e., sport trainers, physicians). This article reviews compliance among injured athletes in the recovery process by presenting the protection motivation theory, personal investment theory, and models of cognitive appraisal. Also, it reviews key research findings about these three theoretical models. In addition, practical guidelines and specific strategies are offered to sport injury rehabilitation personnel to enhance an athlete's adherence to injury rehabilitation. Sports medicine/injury personnel should educate athletes about their injuries and rehabilitation and increase effective communication and active listening. Also, sports medicine/injury personnel should provide social support and encourage positive beliefs of injured athletes. In addition, coping with pain and setting short term goals help athletes to increase their compliance to programmes of rehabilitation.
\end{abstract}


Rehabilitation from sports injuries: From theory to practice

Athletic participation often results in a musculoskeletal injury (1). In the United Kingdom, sport and exercise was the single leading source of injury, accounting for approximately $33 \%$ of all injuries (2). In the United States, child and adult participants in sport and recreation sustain an estimated three to 17 million injuries annually $(3,4)$. As a result, athletes seek medical assistance from physicians, physiotherapists, and sport trainers. For these sports medicine/injury rehabilitation personnel, the goal is to return injured athletes to competition as safely and quickly as possible. The success of sport injury rehabilitation programmes is contingent on following the prescribed protocol (5). Accordingly, compliance with injury rehabilitation programmes has emerged as an area of interest in sports medicine and sport psychology. The aim of this article is to present theories and models of the sport injury rehabilitation adherence literature and discuss the application of these in rehabilitation settings by identifying evidenced-based strategies.

Adherence to sport rehabilitation refers to an injured athlete complying (or not) to a sports medicine/injury personnel's (i.e., physiotherapist, physician, sport trainer) instructions on participation in a rehabilitation programme. The majority of the research in this area has used prospective research designs to investigate the predicted factors associated with injured athletes' compliance to their rehabilitation (6). Three theoretical approaches have been applied to sport injury rehabilitation adherence: (a) protection motivation theory $(7,8,9)$, (b) personal investment theory (10), and (c) cognitive appraisal models $(11,12)$. This article reviews research findings on sport injury rehabilitation adherence in the context of these theoretical approaches and offers strategies for enhancing sport injury rehabilitation adherence based on the theories.

Theory and research on sport injury rehabilitation adherence

\section{Protection motivation theory}


Protection motivation theory is a value expectancy approach that has been applied to athletic injury rehabilitation adherence. According to this theory two cognitive processes are involved in the decision to adopt protective health behaviours (or, alternatively, produce a maladaptive response): the threat appraisal process and the coping appraisal process $(7,8,9)$. The threat appraisal process involves a perception of the severity of a potentially harmful situation (e.g., patients' perceptions of how severe the threat to their health is), and a perceived vulnerability or susceptibility to harm (e.g., how susceptible patients are to the health threat). The coping appraisal process involves a perception of how likely a particular course of action reduces or prevents the threat (labelled response efficacy; for example how patients' response will be effective), and a perception of how likely one can perform particular actions (labelled self-efficacy; for example patients' perceptions of their abilities to follow the recommended rehabilitation programme). Threat appraisal and coping appraisal combine to form patients' protection motivation, which in turn, could direct behavioural responses including explicit behavior (e.g., complete rehabilitation) and inhibition of actions (e.g., avoid exercises that increase the risk of re-injury) (13).

Taylor and May (14) examined the utility of protection motivation theory in predicting adherence to sport injury rehabilitation. They reported that athletes with higher perceptions of susceptibility to re-injury, stronger beliefs in their ability to complete the prescribed modalities, and greater expectancy in the benefits of rehabilitation were more likely to adhere to their rehabilitation programme. Brewer et al. (15) found that treatment efficacy, athletes' self-efficacy, and susceptibility were strongly associated with adherence to a rehabilitation programme. Furthermore, positive associations between belief in the efficacy of treatment and rehabilitation adherence have been reported by Duda et al. (16) and Noyes et al. (17), and this suggests that future research should evaluate the causal contribution of protection motivation theory to adherence in sport injury rehabilitation programmes. The existence of cause and 
effect relationships between protection motivation theory and adherence rehabilitation might contribute to the use of appropriate interventions in order to increase adherence to rehabilitation protocols.

In summary, protection motivation theory can predict adherence behavior. Adherence to a given sport injury treatment is highest when athletes perceive the health threat (and their susceptibility to the threat) as high, their rehabilitation programme to be effective and themselves as able to complete the rehabilitation programme. However, this theory does not account for habitual behaviors, nor does it include a role for social and environmental factors.

\section{Personal investment theory}

Personal investment theory proposes that motivation in specific situations is determined by personal incentives, sense of self-belief, and perceived options (10). Personal incentives are a person's subjective goals for involvement in a particular activity, and these include task incentives, ego incentives, social incentives, and extrinsic incentives. Sense of self-belief includes a person's thoughts and feelings regarding their existence (i.e., the individual's perceptions of competence and self-reliance, one's tendency to behave in accordance with personal goals, one's sense of relationship with significant others), while perceived options are the perceived alternative behaviors which can determine motivation in specific situations.

Duda et al. (16) examined the relationship between personal incentives, sense of selfbeliefs, perceived options, and adherence behavior among 40 injured athletes. They found that personal sport incentives were less important predictors of adherence behaviors than the other two components of personal investment theory. Duda et al. also found that only one personal incentive (i.e., task involvement), and five sense of beliefs (i.e., social support, trait confidence, self-motivation, internal locus of control for rehabilitation, and perceived physical ability) were associated with adherence measures. In addition, the same researchers reported that four perceived option variables (i.e., belief in efficacy of treatment, knowledge of 
treatment, plans for future sport activity, and perceived team role since injury) were related to adherence behavior to injury rehabilitation programme. Athletes who were less self-motivated and placed less emphasis on task incentives were more likely to miss scheduled appointments and less likely to adhere to their rehabilitation programme. Fields et al. (18) identified that self-motivation contributed to adherent or nonadherent behavior during sport injury rehabilitation programmes. Self-motivated injured athletes were less likely to be deterred by outside factors that may have prevented attending rehabilitation sessions. Similarly, Fisher et al. (5) found that injured adherents reported greater perseverance in their rehabilitation than non-adherents. Also, injured athletes who receive social support are more likely to adhere to their rehabilitation programme than the athletes who receive less support $(5,19)$. Future research should investigate the impact of gender, type of sport, and type of injury on adherence motivation using more homogenous samples with regard to the type of injury (16).

In summary, personal investment theory proposes that the meaning of the injury rehabilitation process is determined by individual characteristics and situational factors. It is this subjective interpretation of meaning that influences personal investment, and in turn, behavior. In other words, the meaning of the injury rehabilitation process can influence whether the athlete will choose to adhere to a rehabilitation programme or not.

\section{Models of cognitive appraisal}

Models of cognitive appraisal consider post-injury behavior to be influenced by emotional responses to sport injury, which are thought to occur by the interaction of personal and situational factors $(11,12)$. Within this framework, cognitive appraisal is the process of categorizing an encounter (i.e., an injury) with respect to its significance for well-being (20). These models suggest that an athlete's behavioral response to a sports injury is influenced by the appraisal process (both primary and secondary appraisal), which leads to an emotional response (20). Whereas primary appraisal is concerned with whether an injury is a threat to an 
athlete's well-being and goals, secondary appraisal is concerned with whether the athlete has the resources to cope with the injury.

Research in this area has suggested that athletes who make negative appraisals of their injuries will tend to experience emotional disturbance which can negatively influence their adherence to programmes of rehabilitation. In particular, Brickner (21) suggested that mood disturbance was related inversely to sport injury rehabilitation adherence, while Daly et al. (22) found that cognitive appraisal was correlated with emotional disturbance, and emotional disturbance was inversely related to attendance at rehabilitation sessions.

Cognitive appraisals are influenced by the interaction of personal and situational factors (23). Personal factors are stable, dispositional characteristics and those associated with adherence are self-motivation $(24,14,18)$, pain tolerance $(19,18)$, and task difficulty $(16)$. Situational variables are essentially the athlete's perceptions of the rehabilitation context, and are thought to interact with personal factors that influence cognitive, emotional, and behavioral responses to sport injury (15). These include the belief in the efficacy of the treatment $(16,14)$, comfort of the clinical environment $(5,18)$, convenience of the rehabilitation scheduling $(5,18)$, perceived exertion $(18)$, importance of the rehabilitation to the patient (14), perceived injury severity (14), social support for rehabilitation $(16,19)$, and rehabilitation practitioner expectancy of patient adherence (14).

Another important factor that can predict athletes' compliance to sport injury rehabilitation is the sports medicine/injury practitioner's expectations about an athlete's recovery. These expectations can influence an athlete's cognitive and emotional responses to their injury (25). Positive behaviour towards an athlete's adherence can encourage them not to be nervous, angry, unhappy, disappointed, and impatient during rehabilitation. Consequently, sports medicine/injury rehabilitation personnel's own appraisal of an athlete's adherence can influence athlete's appraisal about treatment (26), and as a result, can increase an athlete's 
rehabilitation treatment attendance. When athletes comply with their prescribed rehabilitation, it is also likely that sports medicine/injury rehabilitation personnel will be more energetic, more helpful, and offer more personal attention (5). This, in turn, can motivate athletes to be more enthusiastic and put more effort towards completing the prescribed exercise programme, and appraise their ability to cope greater. Future research may help identify the importance of this process and the extent to which it has an impact on rehabilitation.

Cognitive appraisal models have a central role in determining athletes' interpretation of their injuries, as well as adherence behavior to their rehabilitation. Both cognitive and behavioral factors are posited to have a direct effect on the way injured athletes appraise their injury and the rehabilitation process. Thus, these factors are associated with behavioral responses such as adherence to an injury rehabilitation programme.

\section{Enhancing sport injury rehabilitation adherence}

Adherence to rehabilitation necessitates a partnership in which sports medicine/injury rehabilitation personnel and athletes collaborate to produce desired end-results. Sometimes athletes find it difficult to comply with prescribed treatments because they encounter cognitive issues (e.g., athletes need to understand the nature of injury, the goals of prescribed treatment, and prognosis for recovery), emotional issues (e.g., athletes need to deal with emotional problems, i.e. anxiety, blame, guilt, anger), and/or behavioral issues (e.g., athletes need to do something about their condition. Sports medicine/injury rehabilitation personnel have to apply their rehabilitation skills in addition to responding to anxieties about treatment and its potential for success. This section offers some practical strategies that can enhance the adherence behavior of injured athletes. These strategies are considered to affect athletes' responses to their injury, and perhaps play a significant role in determining these responses (e.g., complying with or avoiding rehabilitation treatment). Sports medicine/injury rehabilitation personnel can help athletes realize that their treatment is effective and they are 
able to complete their rehabilitation programme by changing the way they appraise their injury $(27,28)$.

Primarily, sports medicine/injury rehabilitation personnel should design rehabilitation programmes for each patient depending upon their individual characteristics. The progress of rehabilitation should be monitored by noting all improvements to the injured athlete. For example, athletes can be informed of any increases in muscle strength by providing them with printed results of isokinetic testing. These results are tangible and visible to athletes, and can help motivate them via aspects of protection motivation and personal investment theories. For example, if an athlete is putting-in extra effort and has high pain tolerance, the rehabilitation programme can be made more complex with an increase in intensity. In this case, sports medicine/injury rehabilitation personnel should try to concurrently increase an athlete's selfconfidence and self-efficacy about completing the programme. Otherwise the intensity of the programme may need to increase slowly and gradually.

\section{Education}

Educating injured athletes about their circumstances as a result of their injury is an important initial step in the rehabilitation process $(27,28,29)$. According to personal investment theory, athletes need to have good knowledge of treatment in order to believe in its efficacy. When athletes are educated about the nature of injury, goals of prescribed treatment, and prognosis for recovery, they better understand how their injury can impact their personal goals. Athletes who appraise their injury as a potential threat to their career often have higher adherence rates to their rehabilitation.

Sports medicine/injury rehabilitation personnel, using principles from protection motivation theory (i.e., the severity of the injury threat and the perceived vulnerability or susceptibility to the injury threat) can inform athletes of the importance of a rehabilitation programme. Giving information about the rehabilitation methods, reasons for doing any 
specific exercise, likelihood of pain, and the effort needed to fully rehabilitate, athletes' feelings their commitment to the rehabilitation programme can be enhanced. Sports medicine/injury rehabilitation practitioners, therefore, need to give specific and clear information about the process of rehabilitation using oral and written material in combination. Asking questions to injured athletes or asking them to restate information given can help in understanding how much the athletes know about the rehabilitation process (e.g., do you understand the severity of your injury, the aim of the rehabilitation programme, why the duration of the rehabilitation programme is two months) (27). Sports medicine/injury rehabilitation personnel can also say to athletes something along the lines of: "Now that you are aware of the situation, you will need to put in extra effort in completing your rehabilitation programme". Failure to offer needed and expected information can also affect an individual's motivation to complete the rehabilitation programme. Sports medicine/injury rehabilitation personnel can inform athletes how much control over their injury they gain by adhering to a rehabilitation programme by suggesting to them: "By adhering to your rehabilitation programme and increasing your muscle strength, muscle endurance, and functional stability, you would be able to return to competing in your sport as soon as possible”.

According to cognitive appraisal models, primary and secondary appraisal impact on the cognitive evaluation of the injury rehabilitation process prompting the development of certain discrete emotions. In particular, athletes' negative interpretations about the injury rehabilitation process may cause emotional disturbances, which can influence their intention to adhere to a rehabilitation programme. Furthermore, some injured athletes may have emotional disturbances because they consider their injury a severe threat to their sport career. Thus, their initial response to an injury can be one of denial, fear, anger, frustration, and depression $(30,31,32)$. Such athletes might feel pessimistic and that nothing can be done to 
change this stressful situation. Therefore, sports medicine/injury rehabilitation personnel should explain to athletes that it is normal to have these feelings, encourage them to express them (27) and assist them to reappraise the injury so that the emotional response to be less extreme.

\section{Communication and listening}

According to personal investment theory, especially the element of the sense of self-belief which includes the sense of relationship with significant others, the athletes' relationship with their sport rehabilitation practitioner can influence their motivation to adhere to a rehabilitation programme. Effective communication between sports medicine/injury rehabilitation personnel and athletes is one way to specifically enhance rehabilitation adherence. Statements such as "You are going to overcome your injury, and I am going to help you in order to succeed it" or "Together we will overcome this injury and will prevent re-injury" by sports medicine/injury rehabilitation personnel clearly encourages in this regard.

Active listening without giving advice or making judgments is an important step in building rapport with an athlete (29). According to protection motivation theory, the sports medicine/injury rehabilitation personnel should listen carefully to injured athletes' perceptions of how severe the injury is to their sport career and how susceptible are to this type of injury. The sports medicine/injury rehabilitation personnel should explain that following the prescribed rehabilitation programme, injury is going to be overcome and not be a threat to the athlete's career. When an athlete asks a question such as "How long am I going to be out?" an appropriate response might be the question, "How hard are you prepared to work at your rehabilitation programme?" Such a response indicates to the athlete that they have to put in effort to complete the rehabilitation and that they are in control of the situation (28).

\section{Social support}


Adherence to sport rehabilitation can be enhanced through social support, the essence of which is listening, encouraging, and caring (34). Social support within the context of rehabilitation is a sense of a self-belief that supports the personal investment theory. Also, according to cognitive appraisal models, social support is a situational variable that is positively related to rehabilitation adherence. Social support is thought to interact with personal factors in order to influence cognitive, emotional, and behavioral responses to sport injury. Methods of social support might also influence the cognitive processes of the protection motivation theory (i.e., treat and coping appraisal processes) in the decision to adhere to a rehabilitation programme. Athletes might realize that they are not vulnerable to the injury and are able to complete the rehabilitation programme. Therefore, adherence to rehabilitation programme should be increased if significant others (e.g., trainer, physiotherapist, teammates, friends, parents) support athletes through the injury, and increase their belief in themselves.

According to personal investment theory's element of the sense of self-beliefs, and to the cognitive appraisal models minimizing the physical or psychological distance between injured athletes and their sport environment, may be a method which can enhance adherence (27). During rehabilitation the athlete might visit a sporting field and watch a match. Injured athletes could also use such opportunities to discuss with their teammates plans for future sport games. In this way, athletes can feel that they are still part of their team. Another method of providing social support is to put the athlete in touch with successfully rehabilitated athletes, especially those who have had similar injuries. For example, sports medicine/injury rehabilitation personnel can arrange for athletes who have successfully completed similar rehabilitation programmes to talk with other injured athlete, or injured athletes can observe other athletes with the same injury completing rehabilitation exercises $(28,29,35)$. Small groups of athletes who have the opportunity to discuss their difficulties 
and successes in rehabilitation can provide others with solutions of how to cope (36).

Transporting equipment to the practice area for on-site rehabilitation allows them to rehabilitate within the context of their sport. This method of social support makes athletes feel that they are still part of the team and increased affiliation serves as motivation to return to the sport (24).

\section{Thought Stoppage}

According to the cognitive appraisal model, injury could be considered a threat to an athlete's well-being and an athlete's goals during the primary appraisal. Thus, athletes who have negative cognitive appraisals of an injury tend to have negative thoughts which can lead to emotional disturbance, and subsequently, possible non-compliance to rehabilitation programmes. Also, according to the threat appraisal process of the protection motivation theory, athletes might appreciate their injury as a threat to their career in sport, appreciating their own vulnerablities. As a result, they might start having negative thoughts, such as "The severity of my injury is high", and "I am vulnerable to this threat". These thoughts can influence their intention to adhere to rehabilitation programme. Therefore, sports medicine/injury rehabilitation personnel, knowing the aforementioned theories, could encourage athletes to use techniques such as thought stoppage (25).

When using thought stoppage, initially athletes need to recognize that they experience negative thoughts about injury and then subsequently can say, "Stop" $(24,37)$. The athlete can then substitute any negative feelings to positive ones by saying statements such as: "I am going to complete the recovery process, I am improving, I am going to return to sport", "I want to return to play again this season", and "This injury is just a minor threat to my career in sport". This can prevent the effect of negative thoughts on the recovery process.

\section{Enhance athletes' beliefs}


According to personal investment theory, beliefs about the meaning of the injury rehabilitation process can determine whether an athlete complies or not with a rehabilitation programme. Athletes need to have a positive attitude towards their rehabilitation programme, and be encouraged to show perseverance in their rehabilitation regimen (27). Sports medicine/injury rehabilitation personnel can enhance athletes' coping appraisal process of the protection motivation theory by increasing athletes' optimism, beliefs, and expectations about the recovery success. By enhancing athletes' beliefs practitioners can also increase athletes' resources to cope with the injury, and thus, facilitate the appraisal process. For example, statements by sports medicine/injury rehabilitation personnel such as "Your balance has been improved by performing the stability exercises in the mini trampoline" or "By completing your cycling exercises your cardiovascular endurance has been improved" can increase an athletes' confidence in the success of their rehabilitation. These statements can help athletes realize their ability to follow recommended rehabilitation programmes and control their injuries. Sports medicine/injury rehabilitation personnel can explain to athletes that their specific rehabilitation programme is the most appropriate for their injury and how the rehabilitation process is also able to prevent or reduce re-injury.

\section{Using short-term goals}

Goal setting operates as a motivational mechanism to influence the degree of effort in striving toward a goal, increase the focusing and directing of attention, and enhance persistence (38). Goal setting has been used in sport settings to enhance performance, perceptions of success, and self-efficacy $(39,40,41)$, and also as been recommended for use within injury rehabilitation $(42,15,43)$. Realistically achievable goals create positive expectations and beliefs about goal attainment, thereby enhancing motivation and adherence to the rehabilitation programme (27). Goal intentions are influenced by beliefs about selfefficacy, and if athletes believe that they can complete their prescribed rehabilitation 
programme, then they are more likely to employ specific and short-term goals. According to the coping appraisal process of protection motivation theory and the secondary cognitive appraisal model, goal setting could help athletes believe they are able to overcome the injury and return to competition successfully.

Sports medicine/injury rehabilitation personnel can employ elements of personal investment theory to enhance athlete's subjective goals in order to increase self-motivation. Particularly, practitioners can support the integration of short-term goals by encouraging athletes to use the present tense in their discussions about rehabilitation (e.g., "What are the aims of my rehabilitation programme this week?", "What rehabilitation exercises do I have to follow today?"). Sports medicine/injury rehabilitation personnel can also monitor goal progress and inform athletes if they have accomplished the rehabilitation goals (or not). Athletes should not set too many goals early in their rehabilitation, as not achieving goals can have a detrimental effect on motivation. Examples of specific short-term goals may include "five degrees increase in knee flexion by the end of the week", and "balance on BAPS board for 30 seconds". Sports medicine/injury rehabilitation personnel should also encourage athletes to set some long-term goals for future sport activity (e.g., "I want to reach my fullfitness level by May" or "In order to get back to sport field in May, I have to put $100 \%$ of my effort in doing my rehabilitation exercises").

\section{Enhancing pain tolerance}

Pain is an emotional and sensory experience which plays an integral part of the athletic experience both in sport performance and in injury (44). Pain tolerance is a personal variable that has been related positively to rehabilitation adherence. Pain tolerance is thought to interact with situational factors in order to influence cognitive, emotional, and behavioral responses to sport injury. Athletes can cease adhering to their rehabilitation, and thus can terminate their programme, due to the belief that they cannot tolerate the pain experienced. 
Pain tolerance depends on an athlete's ability to manage pain. Fisher et al. (5) found that adherents to a rehabilitation programme tolerated pain and discomfort better than nonadherents. Sports medicine/injury rehabilitation personnel and athletes both agree that the appraisal of pain and subsequent focusing on attention are important factors in maintaining rehabilitation adherence (28). Practitioners might wish to first explain to athletes the nature of the pain that they may encounter. Duda et al. (16) and Fisher et al. (5) found that the more knowledge athletes were given about the likelihood of pain, the greater the tolerance to pain was and more likely the athlete adhered to the rehabilitation.

Pain increases muscle tension that restricts blood flow (45). Therefore, applying relaxation techniques such as progressive relaxation (46) can increase comfort and reduce pain. Properly trained sports medicine/injury rehabilitation personnel could also use deep breathing techniques in order to reduce athletes' anxieties and fears associated with pain (45). After relaxation athletes can also be taught imagery, which has been found to be effective in reducing pain in medical and sport settings (47).

Because athletes' perceptions of pain can be altered, they can be helped to cope better with any discomfort by using both dissociation and association strategies. Dissociation is a cognitive strategy, in which injured athletes' attention can be directed away from the pain. Association is the opposite method to dissociation, in which an athlete focuses directly on the specific locus of pain and tries to frame it (48).

\section{Conclusions}

This article applied health promotion theory to the sport injury rehabilitation in order to offer practical approaches to improve performance in practice. Considerable research has been conducted on the factors that predict an athlete's compliance to the process of rehabilitation. During rehabilitation programmes, sports medicine/injury rehabilitation personnel might discuss the psychological affects of injury concurrently with the physical 
aspects of rehabilitation so as to maximally benefit the psychological and physical rehabilitation processes. Having knowledge about goal setting, using positive and effective communication, and understanding athletes' motivation, sports medicine/injury rehabilitation personnel can assist athletes in adhering to their injury rehabilitation. 


\section{References}

1. Kraus JF. Conroy C. Mortality and morbidity from injury from injury and sports and recreation. Ann Rev of Public Health 1984; 5: 163-192.

2. Uitenbroek DG. Sports, exercise, and other causes of injuries: Results of a population survey. Res Q Exerc Sport 1996; 67: 380-385.

3. Bijur PE, Trumble A, Harel Y, Overpeck MD, Jones D, Scheidt, PC. Sports and recreation injuries in U.S. children and adolescents. Arch Ped Adol Med 1995; 149: 1009-1016.

4. Booth W. Arthritis institute tackles sports. Sci 1987; 237: 846-847.

5. Fisher AC, Domn MA, Wuest DA. Adherence to sports-injury rehabilitation programs. Phys Sportsmed 1988; 16: 47-52.

6. Brewer BW. Adherence to sport injury rehabilitation regimens. In: Bull S, editor. Adherence issues in sport and exercise. West Sussex: John Wiley \& Sons, Ltd, 1999. p. $145-68$.

7. Maddux JE, Rogers RW. Protection motivation and self-efficacy: A revised theory of fear appeals and attitude change. J Exper Social Psychol 1983; 19: 469-79.

8. Rogers RW. A protection motivation theory of fear appeals and attitude change. $J$ Psychol 1975; 91: 93-114.

9. Rogers RW. Cognitive and psychological processes in attitude change: A revised theory of protection motivation. In: Cacioppo J, Petty R, editors. Social psychophysiology. New York: Guilford press, 1983. p. 153-76.

10. Maehr M, Braskamp L, editors. The motivation factor: A theory of personal investment. Lexington MA, IN: Lexington Books, 1986.

11. Wiese-Bjornstal DM, Smith AM, LaMott EE. A model of psychologic response to athletic injury and rehabilitation. Athl Train: Sports Health Care Persp 1995; 1 17-30. 
12. Wiese-Bjornstal DM, Smith AM, Shaffer SM, Morrey MA. An integrated model of response to sport injury: Psychological and sociological dynamics. J Appl Sport Psychol 1998; 10: 46-69.

13. Prentice-Dunn S, Rogers RW. Protection motivation theory and preventive health: Beyond the health belief model. Health Educ Res 1986; 1: 153-161.

14. Taylor AH, May S. Threat and coping appraisal as determinants of compliance to sports injury rehabilitation: An application of protection motivation theory. J Sports Sci 1996; 14: 471-782.

15. Brewer BW, Cornelius AE, Van Raalte JL., Petitpas A.J, Sklar JH, et al. Protection motivation theory and adherence to sport injury rehabilitation revisited. Sport Psychol 2003; 17: 95-103.

16. Duda JL, Smart AE, Tappe MK. Predictors of adherence in the rehabilitation of athletic injuries: An application of personal investment theory. J Sport Exer Psychol 1989; 11: $367-81$.

17. Noyes FR, Matthews DS, Mooar PA, Grood ES. The symptomatic anterior cruciate deficient knee. Part II: The results of rehabilitation, activity modification, and counseling on functional disability. J Bone Joint Surg 1983; 65: 163-174.

18. Fields J, Murphey M, Horodyski M, Stopka, C. Factors associated with adherence to sport injury rehabilitation in college - age recreational athletes. $J$ Sport Rehabil 1995; 4: $172-80$.

19. Byerly PN, Worrell T, Gahimer J, Domholdt E. Rehabilitation compliance in an athletic training environment. $J$ Athl Train 1994; 29: 352-55.

20. Lazarus RS, Folkman S, editors. Stress, appraisal, and coping. New York, IN: Springer, 1984. 
21. Brickner JC. Mood states and compliance of patients with orthopedic rehabilitation [dissertation]. Springfield College, MA, 1997.

22. Daly JM, Brewer BW, Van Raalte JL, Petitpas AJ, Sclar JH. Cognitive appraisal, emotional adjustment, and adherence to rehabilitation following knee surgery. J Sport Rehabil 1995; 4: 23-30.

23. Brewer BW. Psychology of sport injury rehabilitation. In: Singer RN, Housenblas A, Janelle JM, editors. Handbook of sport psychology. New York: Wiley, 2001. p. 789809.

24. Brewer BW, Van Raalte JL, Cornelius AE, Petitpas AJ, Sklar JH, Pohlman MH, et al. Psychological factors, rehabilitation adherence, and rehabilitation outcome following anterior cruciate ligament reconstruction. Rehabil Psychol 2000; 45: 20-37.

25. Wilder KC. Clinician's expectations and their impact on an athlete's compliance in rehabilitation. J Sport Rehabil 1994; 3: 168-75.

26. Ievleva L, Orlick T. Mental links to enhanced healing: An exploratory study. Sport Psychol 1991; 5: 25-40.

27. Fisher AC. Counseling for improved rehabilitation adherence. In: Ray R, WieseBjornstal DM, editors. Counseling in sports medicine. United States: Human Kinetics, 1999. p. 275-291.

28. Fisher AC, Scriber KC, Matheny ML, Alderman MH, Bitting LA. Enhancing athletic injury rehabilitation adherence. J Athl Train 1993; 28: 312-318.

29. Gordon S, Milios D, Grove JR. Psychological aspects of the recovery process from sport injury: the perspective of sport physiotherapists. Aust J Sci Med Sport 1991; 23: 53-60.

30. Brewer BW, Jeffers EK, Petitpas JA, Van Raalte JL. Perceptions of psychological interventions in the context of sport injury rehabilitation. Sport Psychol 1994; 8: 176-88. 
31. Leddy MH, Lambert MJ, Ogles BM. Psychological consequences of athletic injury among high-level competitors. Res Q Exerc Sport 1994; 65: 347-54.

32. Tracey J. The emotional response to the injury and rehabilitation process. $J$ Appl Sport Psychol 2003; 15: 279-93.

33. Udry E, Gould D, Bridges D, Beck L. Down but not out: Athlete responses to seasonending injuries. J Sport Exerc Psychol 1997; 17: 229-48.

34. Caplan RD, Robinson EAR, French JRP, Caldewell JR, Shinn M. Adhering to Medical Regimens: Pilot Experiments in Patient Education and Social Support [dissertation]. Ann Arbor Mich, IN: Institute for Social Research, University of Michigan, 1976.

35. Worrell TW. The use of behavioral and cognitive techniques to facilitate achievement of rehabilitation goals. J Sport Rehabil 1992; 1: 69-75.

36. Weiss MR, Troxel RK. Psychology of the injured athlete. Athl Train 1986; 24: 104-110.

37. Gieck J. Psychological considerations of rehabilitation. In: Prentice WE, editor. Rehabilitation techniques in sports medicine. St. Louis: Times Mirror/ Mosby, 1990. p. 107-122.

38. Locke EA, Latham GP. A Theory of Goal Setting and Task Performance. New Jersey, IN: Prentice-Hall Eaglewood Cliffs, 1990.

39. Filby WCD, Maynard IW, Graydon JK. The effect of multiple-goal strategies on performance outcomes in training and competition. J Appl Sport Psychol 1999; 11: 230246.

40. Kyllo BL, Landers DM. Goal setting in sport and exercise: A research synthesis to resolve the controversy. J Sport Exerc Psychol 1995; 17: 117-132.

41. Swain ABJ, Jones G. Goal attainment scaling: Effects of goal setting interventions on selected subcomponents of basketball performance. Res Q Exerc Sport 1995; 66: 51-63. 
42. Weinberg R, Stichers T, Richardson P. Effects of a seasonal goal-setting program on lacrosse performance. Sport Psychol 1994; 8: 166-175.

43. Evans L, Hardy L. Injury rehabilitation: A goal setting intervention study. ResQ Exerc Sport 2002; 73: 310-319.

44. International Association for the Study of Pain. Classification of chronic pain: descriptions of chronic syndromes and definitions of pain terms. Pain 1986; 27: S1S225.

45. Cousin MJ, Philips GD. Acute pain management. In: Cousin MJ, Philips GD, editors. Clinics in critical care medicine. New York: Churchill, 1985. p. 82-117.

46. Whitmarsh BG, Alderman RB. Role of psychological skills training in increasing athletic pain tolerance. Sport Psychol 1993; 7: 388-99.

47. Whitmarsh BG, Alderman RB. Role of psychological skills training in increasing athletic pain tolerance. Sport Psychol 1993; 7: 388-99.

48. Nideffer RM. The injured athlete: Psychological factors in treatment. Orthop Clin North Am 1983; 14: 372-85. 